\title{
Assessment of 17 clinically available renal biomarkers to predict acute kidney injury in critically ill patients
}

Yating $\mathrm{Hou}^{1,2+}$, Yujun Deng ${ }^{3+}$, Linhui $\mathrm{Hu}^{4}$, Linling $\mathrm{He}^{3}$, Fen $\mathrm{Yao}^{3}$, Yifan Wang ${ }^{3}$, Jia Deng $^{3}$, Jing $\mathrm{Xu}^{3}$, Yirong $\mathrm{Wang}^{3}$, Feng $\mathrm{Xu}^{5}$, Chunbo $\mathrm{Chen}^{3,4,6}$

'Department of Clinical Research Center, Maoming People's Hospital, Maoming 525000, Guangdong Province, China;

${ }^{2}$ Department of Oncology, Maoming People's Hospital, Maoming 525000, Guangdong Province, China; ${ }^{3}$ Department of Critical Care Medicine, Guangdong Provincial People's Hospital, Guangdong Academy of Medical Sciences, Guangzhou 510080, Guangdong Province, China;

${ }^{4}$ Department of Critical Care Medicine, Maoming People's Hospital, Maoming 525000, Guangdong, China;

${ }^{5}$ Department of Emergency Medicine, Shandong University Qilu Hospital, Jinan 250012, Shandong Province, China;

${ }^{6}$ The Second School of Clinical Medicine, Southern Medical University, Guangzhou 510080, Guangdong, China.

\section{ABSTRACT}

Background: Systematic estimation of renal biomarkers in the intensive care unit (ICU) patients is lacking. Seventeen biomarkers were assessed to predict acute kidney injury (AKI) after admission to ICU. Materials and methods: A prospective, observational study was conducted in the general ICU of Guangdong Provincial People's Hospital. Seventeen serum or urine biomarkers were studied for their abilities alone or in combination for predicting AKI and severe AKI. Results: Of 1498 patients, 376 (25.1\%) developed AKI. Serum cystatin $\mathrm{C}$ (CysC) showed the best performance for predicting both AKI (area under the receiver operator characteristic curve $[A \cup C]=0.785$, mean square error $[M S E]=0.118$ ) and severe AKI (AUC $=0.883$, MSE $=0.06$ ). Regarding biomarkers combinations, Cys C plus $N$-acetyl- $\beta$ d-glucosaminidase-to-creatinine ratio (NAG/Cr) was the best for predicting $\mathrm{AKI}$ ( $\mathrm{AUC}=0.856$, $\mathrm{MSE}=0.21)$. At the same time, CysC plus lactic acid (LAC) performed the best for predicting severe AKI (AUC $=0.907$, MSE $=0.058$ ). Regarding combinations of biomarkers and clinical markers, CysC plus Acute Physiology and Chronic Health Evaluation (APACHE) II score showed the best performance for predicting AKI $(A \cup C=0.868$, MSE $=0.407)$. In contrast, CysC plus Multiple Organ Dysfunction Score (MODS) had the highest predictive ability for severe AKI (AUC $=0.912$, MSE $=0.488$ ). Conclusion: Apart from CysC, the combination of most clinically available biomarkers or clinical markers does not significantly improve the forecasting ability, and the cost-benefit ratio is not economical.

Key words: acute kidney injury, biomarkers, critically ill adults, cystatin C

\section{INTRODUCTION}

Acute kidney injury (AKI) is a public health burden with a high prevalence of more than $20 \%$ and increasing incidence. ${ }^{[1-4]}$ AKI patients would probably be more vulnerable to worse clinical outcomes, including protracted hospitalization, the requirement for renal replacement therapy (RRT), the progress of new chronic kidney disease (CKD), and an increased risk of mortality. ${ }^{[5,6]}$ Based on the early detection of AKI, timely treatment can rapidly terminate kidney's underlying injury to preserve or even reverse kidney function. ${ }^{[7-9]}$

Though serum creatinine $(\mathrm{sCr})$ and urine output (UO) are the most commonly used markers of renal function at present, they still have the limitation that sCr and UO lag behind actual renal injury. Consequently, physicians are keen on discovering 
biomarkers that could be used to discern, diagnose, or supervise the therapeutic response of AKI on time. ${ }^{[10,11]}$ As a result, diverse urinary and serum proteins and molecules have been meticulously inspected over the past decade as biomarkers for kidney injury. ${ }^{[12-15]}$ Quite a few potential serum and urinary biomarkers of kidney injury have been recognized, including neutrophil gelatinase-associated lipocalin (NGAL), ${ }^{[16]}$ kidney injury molecule-1 (KIM1), ${ }^{[17,18]}$ interleukin-18 (IL-18), ${ }^{[19]}$ urinary angiotensinogen (uAGT) ${ }^{[20,21]}$ urinary matrix metalloproteinase-7 (uMMP7), ${ }^{[20,22,23]}$ tissue inhibitor of metalloproteinase-2 (TIMP-2), and insulin-like growth factor-binding protein (IGFBP). ${ }^{[24]}$

In contrast to the extensive research into the biomarkers mentioned earlier, comprehensive research into clinically available renal biomarkers is far from adequate. Clinically, there are many biomarkers related to renal function, which can be classified into biomarkers chiefly reflecting glomerular filtration (i.e., cystatin $\mathrm{C}[\mathrm{Cys} C]$ ), glomerular integrity (i.e., albumin $[\mathrm{ALB}]$ and albumin-to-creatinine ratio $[\mathrm{ACR}]),{ }^{[15,25]}$ tubular damage (i.e., $N$-acetyl- $\beta$-dglucosaminidase-to-creatinine ratio $[\mathrm{NAG} / \mathrm{Cr}]),{ }^{[26]}$ and inflammation (i.e., interleukin-6 [IL-6]). ${ }^{[27]}$ Moreover, some common clinical factors, such as scoring systems and UO in the first $24 \mathrm{~h}$ after intensive care unit (ICU) admission, are often associated with renal function. Accordingly, these markers can be potential predictors of AKI as they reflect the kidney function theoretically. However, there is still a lack of systematic evaluation of the markers' predictive abilities alone or in combination in critically ill patients. Thus, we tested the concentration of 17 clinically available biomarkers in urine or serum in a large population of 1498 patients admitted to the ICU.

\section{MATERIALS AND METHODS}

\section{Study design and participants}

This prospective observational study was conducted in the general ICU of Guangdong Provincial People's Hospital from January 2017 to December 2017. All the patients in the ICU were eligible for inclusion. Reasons for exclusion were as follows: age under 18 years, preexisting AKI before ICU admission, a history of nephrectomy or end-stage renal disease (ESRD) or renal transplantation, preexisting dialysis before ICU admission, or readmission to ICU. The primary outcome was the development of AKI, defined as detecting any stage of AKI occurring no more than 1 week after ICU admission. The secondary outcome was the development of severe AKI, defined as the combined endpoints of AKI stage 2 or 3 according to the Kidney Disease: Improving Global Outcomes (KDIGO) criteria, ${ }^{[28]}$ no more than 1 week after ICU admission. The study protocol complied with the Strengthening the Reporting of Observational Studies in Epidemiology ${ }^{[29]}$ and the Standards for Reporting
Diagnostic Accuracy criteria. ${ }^{[30]}$ Written informed consent was obtained from all participants before their enrollment. The study protocol was approved by the ethics committee of Guangdong Provincial People's Hospital and adhered to the ethical guidelines stated in the Declaration of Helsinki.

\section{Definitions}

Within 1 week after ICU admission, AKI was diagnosed according to the KDIGO criteria, ${ }^{[28]}$ while severe AKI was defined as KDIGO stage 2 or stage 3. The baseline estimated glomerular filtration rate (eGFR) was estimated by the simplified Modification of Diet in Renal Disease formula. ${ }^{[31]} \mathrm{CKD}$ was defined as baseline eGFR $<60 \mathrm{ml} /$ $\mathrm{min} / 1.73 \mathrm{~m}^{2}$. For baseline $\mathrm{sCr}$, we used the last available sCrwithin the latest 3 months before this hospitalization or the $\mathrm{sCr}$ at hospital admission. ${ }^{[32-34]}$ We adopted $\mathrm{sCr}$ rather than $\mathrm{UO}$ to diagnose AKI, since the UO criteria could be affected by administering diuretics or obesity.

The predictive performances of biomarkers for AKI were stratified into useless, poor, fair, good, and excellent according to the area under the receiver operator characteristic curve (AUC) located in the ranges of $<0.60$, $0.60-0.69,0.70-0.79,0.80-0.89$, and $\geq 0.90$, respectively. ${ }^{[35]}$ Biomarkers with a cutoff value higher than the upper limit of normal range (ULN) were also classified as useless because the present study aims to explore the biomarkers' abilities in predicting the development (onset) rather than the severity, progression, persistence, or survival outcomes of AKI. When the optimal cutoff value is at a higher level than the ULN, even a physician not specializing in renal diseases can intuitively be aware that renal dysfunction (AKI) has already occurred, independent of any prediction.

\section{Sample measurements and data collection}

The blood and urine samples were collected simultaneously within $1 \mathrm{~h}$ after ICU admission. All the candidate biomarkers were tested using a standard protocol by experienced specialists in the central laboratory of Guangdong Provincial People's Hospital. All test methods should not be formally used until quality control. Multiplex assays and enzyme-linked immunosorbent assays were used to test urinary or blood concentrations of biomarkers according to the manufacturer's instructions. Serum biomarkers included blood urea nitrogen (BUN), sCr, CysC, IL-6, lactic acid (LAC), lactate dehydrogenase $(\mathrm{LDH})$, phosphate, procalcitonin (PCT), and superoxide dismutase (SOD). Urinary biomarkers tested included ACR, ALB, alkaline phosphatase (ALP), gamma-glutamyl transpeptidase (GGT), NAG/Cr, total protein (TP), transferrin (TRF), $\alpha 1$-microglobulin $(\alpha 1 \mathrm{M})$, and $\beta 2$-microglobulin $(\beta 2 \mathrm{M})$. Urinary creatinine concentration was used to normalize urinary biomarker measurements to account for urinary concentration on urinary biomarker concentrations. Except 
for sCr testing, all biomarkers were tested once on ICU admission. In addition, sCr testing was performed on ICU admission and at least once daily after admission as a portion of routine clinical care during an ICU stay.

In the meantime, clinical and demographic characteristics were collected immediately once these patients were enrolled in this research. The variables evaluated were: age, sex, body mass index (BMI), preexisting clinical conditions, admission type, baseline sCr, admission sCr, baseline eGFR, admission eGFR, Acute Physiology and Chronic Health Evaluation (APACHE) II score, Multiple Organ Dysfunction Score (MODS), Sequential Organ Failure Assessment (SOFA) score, urine production (UP) for the first $24 \mathrm{~h}$ after admission, and $\mathrm{sCr}$ percent change and the clinical outcomes. We also prospectively recorded the hourly UO from enrollment to ICU discharge.

\section{Statistical analysis}

The statistical analysis was conducted with the software programs SAS (SAS v9.4; SAS Institute, Cary, NC, USA) and R v3.3.3 (R Foundation for Statistical Computing, Vienna, Austria) using RStudio v1.0.136 (RStudio Inc, Boston, MA, USA). For AKI and non-AKI, we used frequencies and percentages for categorical variables and medians and ranges for continuous variables to summarize demographic and clinical baseline characteristics numerically. Using Fisher's exact test or the Wilcoxon rank-sum test, comparisons of patient characteristics between AKI and non-AKI were performed. What is more, we inspected the pairwise associations between biomarkers based on Spearman's rank correlation coefficient. By conducting a Euclidean distance-based unsupervised clustering analysis and constructing a dendrogram to display identified clusters, groups of similar biomarkers were identified. ${ }^{[36]}$

We measured the concentration of 17 urinary and blood analytes to evaluate the capability of the biomarker to predict the occurrence of AKI alone or in combination. Biomarkers were divided into four mechanistic groups (inflammation, oxidative stress, glomerular dysfunction, and tubular dysfunction) according to the presumed pathophysiologic mechanism they reflected. The predictive performance of markers was assessed using simple logistic regression (SLR) models. SLR results were obtained with AUCs and corresponding 95\% confidence intervals (CIs). The analysis was achieved via AUC with a leave-oneout cross-validation approach. A leave-one-out crossvalidation method was used to determine the model's generalization ability without an independent test data set. Therefore, three relevant indicators were generated: the proportion of AKI misclassification, the balance of non-AKI misclassification, and the mean square error (MSE). MSE was an average calculation of the squared difference between observations' event status ( 0 or 1$)$ and the predicted probability of being an event based on the SLR model. Models with highly discriminating markers yield high possibilities for events and low chances for nonevents, resulting in minor differences and small MSE values. For each leave-one-out cross-validated model, AUC was further used to evaluate performance. When the values of AUC were different, markers to predict AKI were prioritized according to the values of AUC. However, when the values of AUC were the same, MSE was compared because lower MSE indicated better fit of the model. The difference between AUCs for detecting a biomarker for AKI and severe AKI was found, and the performance of this biomarker was identified according to the lower AUC if no difference existed.

\section{RESULTS}

\section{Patient characteristics and outcomes}

Figure 1 demonstrates the protocol and flow diagram of the screening process. Among 1761 critically ill adult patients enrolled in the study, 263 were excluded, and therefore, 1498 patients were involved in the study. Twentyfive percent (376/1498) of these patients developed AKI according to the KDIGO criteria. Among them, with the aggravation of AKI severity, the mortality of patients was increasing (Figure 1). There were no differences between the AKI and non-AKI groups based on BMI or preexisting clinical conditions of cancer and thyroid disease. Apart from these, other variables of baseline characteristics and outcomes were different between the AKI and the nonAKI groups (Table 1).

\section{Biomarker characteristics}

Before the prediction analysis, we made two analyses, cluster analysis and correlation analysis, to identify the relationship of individual biomarkers with each other. Foremost, we did an unsupervised cluster analysis to evaluate which biomarkers were similar to each other (Supplementary Figure 1). We discovered several resemblances to our prior grouping of biomarkers. Interestingly, portions of the proteins that we assumed would be analogous to each other were clustered contrarily, such as $\mathrm{Cys} C$ and BUN. We then compared the correlation coefficients for each of the biomarkers using Spearman's rank correlation coefficient (Supplementary Table 1).

\section{Biomarker detection abilities for AKI and severe AKI}

Table 2 displays the predictive features for AKI for each of the 17 analytes classified by the mechanistic biomarker category. Since higher AUC indicated better predictive ability and lower MSE indicated better fit of the model with 


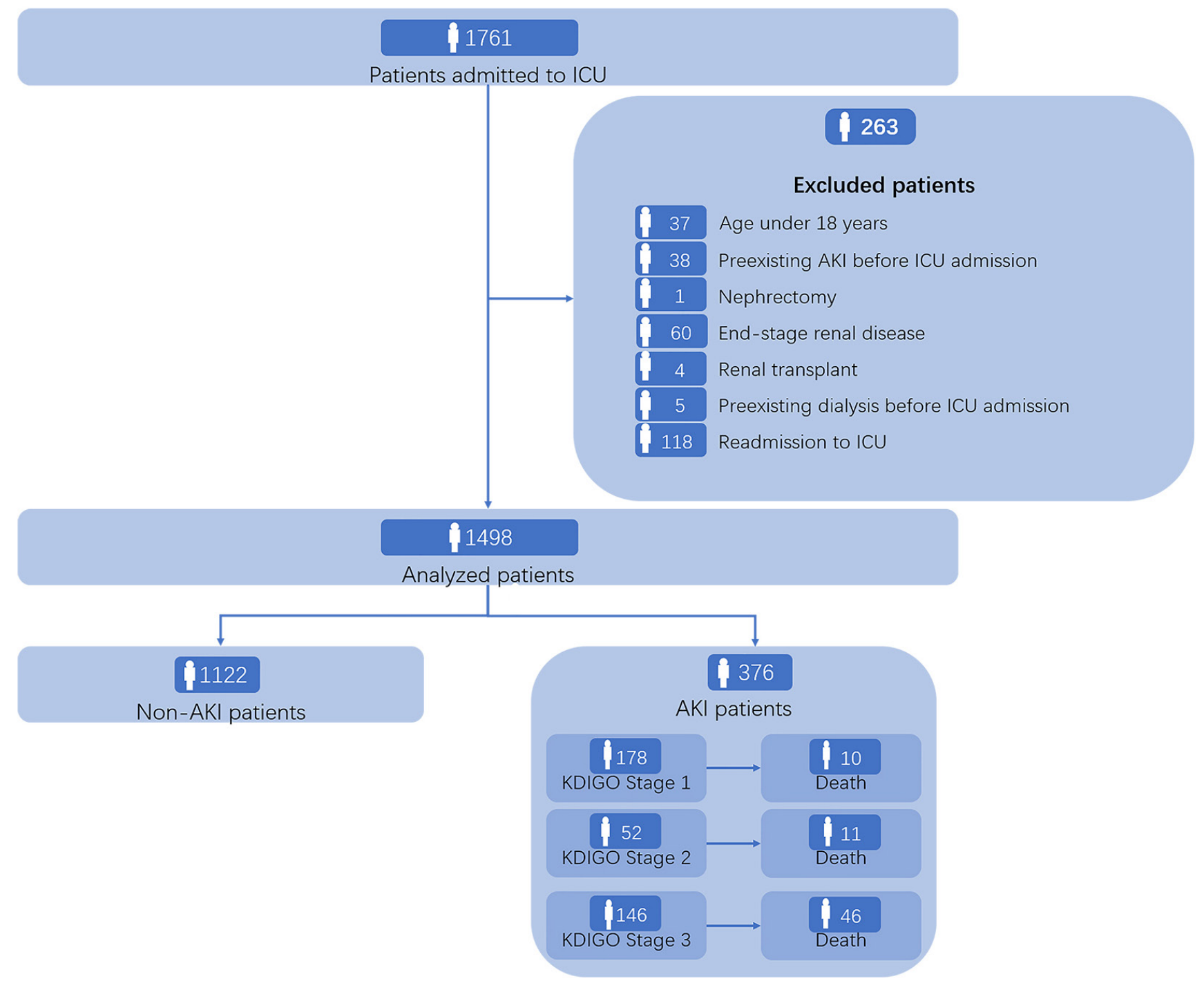

Figure 1: Study flowchart. AKI: acute kidney injury; ICU: intensive care units; KDIGO: Kidney Disease: Improving Global Outcomes.

real world, biomarkers were evaluated for their predictive performance with AUC and MSE. In contrast to all of the biomarkers and clinical markers, $\mathrm{Cys} C$ notably had the highest predictive values for both endpoints (Tables 2 and 3). Among biomarkers, the highest AUC value of 0.785 was seen for CysC and BUN to predict AKI (Table 2). Used in the prediction of AKI, LDH had an AUC of 0.764, and ACR, ALB, IL-6, and NAG/Cr had AUCs of 0.759, $0.727,0.710$, and 0.708 , respectively. For comparison of clinical markers, the APACHE II score, MODS, SOFA score, and eGFR at admission had AUC values of 0.844 , $0.802,0.798$, and 0.703 , respectively.

Of the 17 renal biomarkers, 7 biomarkers (serum biomarkers of PCT, SOD, and phosphate and urinary biomarkers of TP, ALP, GGT, and $\beta 2 \mathrm{M}$ ) with AUC less than 0.6 and 2 biomarkers (serum BUN and urinary ALB) with a fairto-good apparent AUC value but a cutoff value higher than the ULN were useless for both AKI and severe AKI prediction (Tables 2 and 3, Supplementary Table 2). Three biomarkers (serum LAC, urinary TRF, and urinary $\alpha 1 \mathrm{M}$ ) were poor for the prediction of both outcomes. Although urinary TRF had a nominal AUC of 0.764 for severe AKI prediction, there was no statistically significant difference with an AUC of 0.693 for AKI prediction; so, TRF was poor for the prediction of both AKI and severe AKI. The same was true for urinary $\alpha 1 \mathrm{M}$ (Tables 2 and 3). Three biomarkers (serum IL-6, serum LDH, and urinary NAG/ $\mathrm{Cr}$ ) had a fair performance for predicting both outcomes. Two biomarkers (serum CysC and urinary ACR) performed fairly for predicting AKI and showed good performance for severe AKI prediction. These data demonstrated that the 
Hou et al.: Assessment of 17 clinically available renal biomarkers to predict acute kidney injury in critically ill patients

\begin{tabular}{|c|c|c|c|}
\hline Characteristics & Non-AKI $(n=1122)$ & AKI $(n=376)$ & $P$-value \\
\hline \multicolumn{4}{|l|}{ Demographic variables } \\
\hline Age, years & $54.0(44.0-65.0)$ & $62.0(51.0-71.0)$ & $<0.001$ \\
\hline Male sex, $n(\%)$ & $596(53.1)$ & $235(62.5)$ & 0.0021 \\
\hline $\mathrm{BMI}, \mathrm{kg} / \mathrm{m}^{2}$ & $21.9(19.6-24.5)$ & $22.2(19.8-24.8)$ & 0.3245 \\
\hline \multicolumn{4}{|l|}{ Preexisting clinical conditions, $n(\%)$} \\
\hline Hypertension & $197(17.6)$ & $140(36.8)$ & $<0.001$ \\
\hline DM & $83(7.4)$ & $79(20.7)$ & $<0.001$ \\
\hline CKD & $9(0.8)$ & $57(15.0)$ & $<0.001$ \\
\hline Chronic liver disease & $11(1.0)$ & $13(3.4)$ & 0.0011 \\
\hline Stroke & $88(7.9)$ & $71(18.6)$ & $<0.001$ \\
\hline COPD & $18(1.6)$ & $24(6.3)$ & $<0.001$ \\
\hline CAD & $32(2.9)$ & $44(11.6)$ & $<0.001$ \\
\hline $\mathrm{HF}$ & $14(1.3)$ & $41(10.8)$ & $<0.001$ \\
\hline Cancer & 207 (18.5) & $69(18.1)$ & 0.8546 \\
\hline Thyroid disease & $52(4.7)$ & $9(2.5)$ & 0.0505 \\
\hline Sepsis & $20(1.8)$ & $42(11.0)$ & $<0.001$ \\
\hline Admission type, $n(\%)$ & & & $<0.001$ \\
\hline Elective surgery & $876(78.1)$ & $107(28.5)$ & \\
\hline Emergency surgery & $65(5.8)$ & $45(12.0)$ & \\
\hline Medical & $181(16.1)$ & $224(59.6)$ & \\
\hline Baseline serum creatinine, $\mathrm{mg} / \mathrm{dL}$ & $0.77(0.64-0.94)$ & $0.88(0.65-1.57)$ & $<0.001$ \\
\hline Serum creatinine at admission, $\mathrm{mg} / \mathrm{dL}$ & $0.85(0.71-1.02)$ & $1.22(0.80-2.17)$ & $<0.001$ \\
\hline Baseline eGFR, $\mathrm{mL} / \mathrm{min} / 1.73 \mathrm{~m}^{2}$ & $116.83(97.38-139.15)$ & $100.62(51.50-142.42)$ & $<0.0001$ \\
\hline eGFR at admission, $\mathrm{mL} / \mathrm{min} / 1.73 \mathrm{~m}^{2}$ & $104.14(87.58-125.24)$ & 70.07 (33.27-107.52) & $<0.0001$ \\
\hline APACHE II & $6(4-10)$ & $15(10-21)$ & $<0.001$ \\
\hline UP, $\mathrm{mL} / \mathrm{kg} / \mathrm{h}$ & $1.71(1.23-2.26)$ & $1.29(0.69-2.08)$ & $<0.001$ \\
\hline Percentage of change in serum creatinine & $0.05(0-0.15)$ & $0.105(0-0.5)$ & $<0.001$ \\
\hline \multicolumn{4}{|l|}{ Outcomes } \\
\hline Length of ICU stay, days & $2(2-3)$ & $6(3-13)$ & $<0.001$ \\
\hline Length of hospital stay, days & $15(11-21)$ & $20(12-31)$ & $<0.001$ \\
\hline RRT during ICU stay, $n(\%)$ & $13(1.2)$ & $96(26.2)$ & $<0.001$ \\
\hline ICU mortality, $n(\%)$ & $13(1.2)$ & $55(14.4)$ & $<0.001$ \\
\hline In-hospital mortality, $n(\%)$ & $19(1.7)$ & $69(18.1)$ & $<0.001$ \\
\hline
\end{tabular}

The non-normally distributed continuous variables are expressed as median (25th percentile to 75 th percentile IQR). Categorical variables are expressed as $n(\%)$.

AKI, acute kidney injury; APACHE II, Acute Physiology and Chronic Health Evaluation II; BMI, body mass index; CAD, coronary artery disease; CKD, chronic kidney disease; COPD, chronic obstructive pulmonary disease; DM, diabetes mellitus; eGFR, estimated glomerular filtration rate; HF, heart failure; ICU, intensive care unit; IQR, interquartile range; RRT, renal replacement therapy; UP, urine production for the first $24 \mathrm{~h}$ after admission. 
Hou et al.: Assessment of 17 clinically available renal biomarkers to predict acute kidney injury in critically ill patients

Table 2: Predictive characteristics of molecular and clinical markers for total AKI

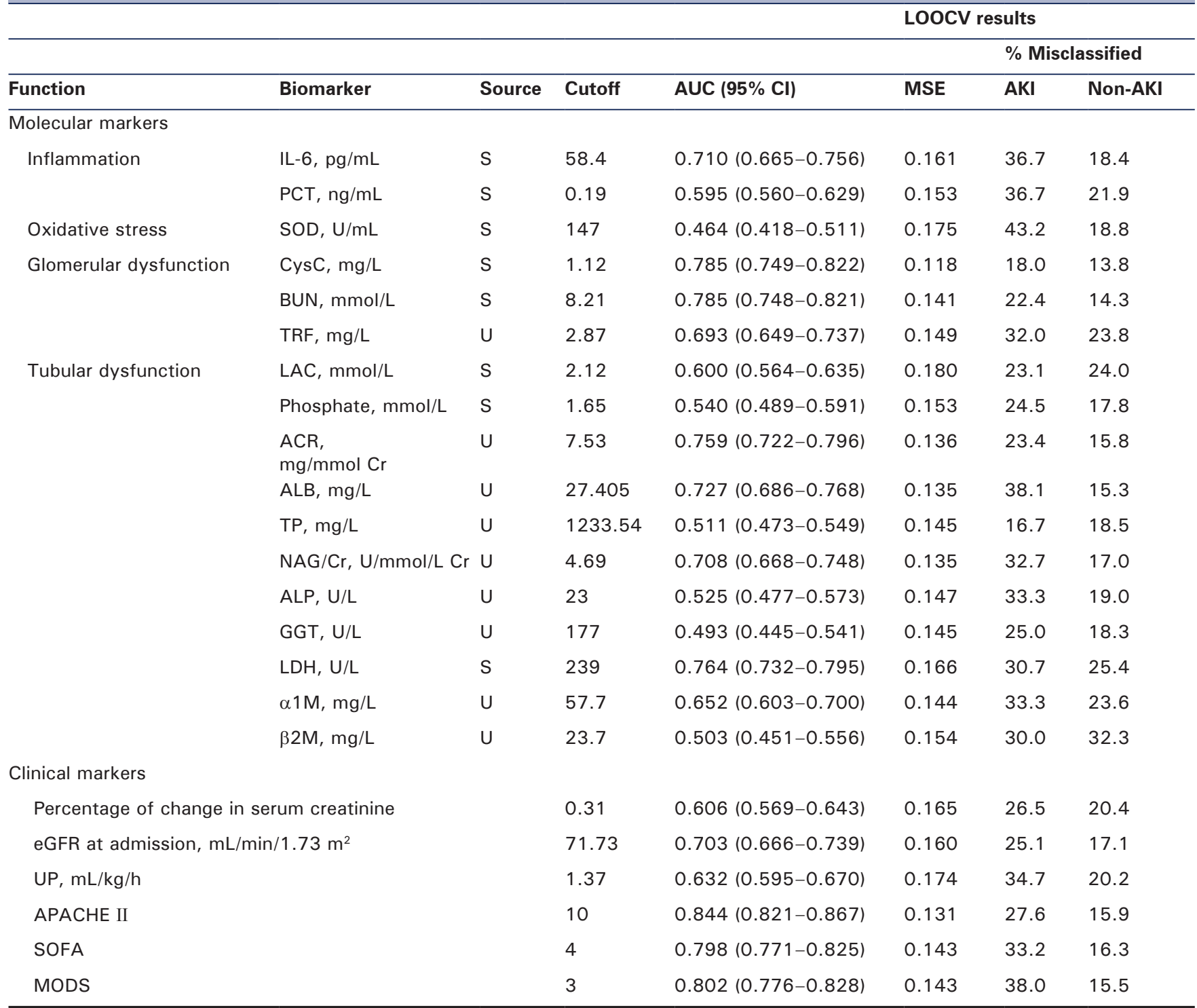

ACR, albumin-to-creatinine ratio; AKI, acute kidney injury; ALB, albumin; ALP, alkaline phosphatase; APACHE II, Acute Physiology and Chronic Health Evaluation II; $\mathrm{AUC}$, area under the receiver operating characteristic curve; $\mathrm{BUN}$, blood urea nitrogen; $\mathrm{Cl}$, confidence interval; $\mathrm{Cr}$, creatinine; CysC, cystatin C; eGFR, estimated glomerular filtration rate; GGT, gamma-glutamyl transpeptidase; IL-6, interleukin-6; LAC, lactic acid; LDH, lactate dehydrogenase; LOOCV, leave-one-out cross-validation; MODS, Multiple Organ Dysfunction Score; MSE, mean square error; NAG, $N$-acetyl- $\beta$-d-glucosaminidase; NAG/ Cr, NAG-to-creatinine ratio; PCT, procalcitonin; S, serum; SOD, superoxide dismutase; SOFA, Sequential Organ Failure Assessment; TP, total protein; TRF, transferrin; $U$, urine; UP, urine production for the first $24 \mathrm{~h}$ after admission; $\alpha 1 \mathrm{M}, \alpha 1$-microglobulin; $\beta 2 \mathrm{M}, \beta 2$-microglobulin.

currently available biomarkers and clinical markers are better predictors for severe AKI than for AKI. The prediction performance of all 17 biomarkers for AKI and severe AKI prediction is summarized in Table 4.

We subsequently compared the abilities of the biomarkers to predict severe AKI. CysC, LDH, APACHE II score, MODS, and SOFA score showed marked improvements in prediction, whereas the predictive ability of many biomarkers was obviously improved (Table 3). CysC was the best predictor of severe AKI, with the highest AUC of 0.883 and the smallest MSE of 0.060 .

\section{Prediction abilities of combinations of biomarkers or clinical markers for AKI and severe AKI}

We evaluated the combinations of biomarkers and clinical markers to predict the two outcomes by ranking groups of biomarkers according to AUC and MSE. The combination of CysC and APACHE II score had the highest AUC and the lowest MSE to predict AKI, while CysC and MODS panel had the highest AUC and a low MSE to predict severe AKI (Table 5, Figure 2). Furthermore, the panel of CysC and NAG/Cr had higher AUC and lower MSE to predict both outcomes. As suggested by our correlation analysis, 
Hou et al.: Assessment of 17 clinically available renal biomarkers to predict acute kidney injury in critically ill patients

Table 3: Predictive characteristics of molecular and clinical markers for severe AKI

\begin{tabular}{|c|c|c|c|c|c|c|c|}
\hline \multirow[b]{3}{*}{ Function } & \multirow[b]{3}{*}{ Biomarker } & \multirow[b]{3}{*}{ Source } & \multirow[b]{3}{*}{ Cutoff } & \multirow[b]{3}{*}{ AUC $(95 \% \mathrm{CI})$} & \multicolumn{3}{|c|}{ LOOCV results } \\
\hline & & & & & \multirow[b]{2}{*}{ MSE } & \multicolumn{2}{|c|}{$\%$ Misclassified } \\
\hline & & & & & & AKI & Non-AKI \\
\hline \multicolumn{8}{|l|}{ Molecular markers } \\
\hline \multirow[t]{2}{*}{ Inflammation } & $\mathrm{IL}-6, \mathrm{pg} / \mathrm{mL}$ & $\mathrm{S}$ & 57.6 & $0.760(0.710-0.809)$ & 0.104 & 47.6 & 11.4 \\
\hline & $\mathrm{PCT}, \mathrm{ng} / \mathrm{mL}$ & $\mathrm{S}$ & 0.26 & $0.619(0.575-0.663)$ & 0.081 & 0.0 & 11.5 \\
\hline Oxidative stress & $\mathrm{SOD}, \mathrm{U} / \mathrm{mL}$ & $\mathrm{S}$ & 147.5 & $0.516(0.449-0.583)$ & 0.101 & 42.9 & 9.5 \\
\hline \multirow[t]{3}{*}{ Glomerular dysfunction } & CysC, mg/L & $\mathrm{S}$ & 1.13 & $0.883(0.850-0.916)$ & 0.060 & 18.0 & 7.4 \\
\hline & $\mathrm{BUN}, \mathrm{mmol} / \mathrm{L}$ & $\mathrm{S}$ & 9.58 & $0.856(0.819-0.893)$ & 0.091 & 30.5 & 7.1 \\
\hline & $\mathrm{TRF}, \mathrm{mg} / \mathrm{L}$ & $U$ & 3.11 & $0.764(0.708-0.820)$ & 0.079 & 30.8 & 11.7 \\
\hline \multirow[t]{11}{*}{ Tubular dysfunction } & $\mathrm{LAC}, \mathrm{mmol} / \mathrm{L}$ & $\mathrm{S}$ & 2.24 & $0.633(0.585-0.681)$ & 0.108 & 43.6 & 11.8 \\
\hline & Phosphate, mmol/L & $\mathrm{S}$ & 1.6 & $0.574(0.502-0.647)$ & 0.087 & 29.0 & 9.2 \\
\hline & $\begin{array}{l}\mathrm{ACR}, \\
\mathrm{mg} / \mathrm{mmol} \mathrm{Cr}\end{array}$ & $U$ & 10.42 & $0.835(0.793-0.877)$ & 0.067 & 25.0 & 7.8 \\
\hline & ALB, mg/L & $U$ & 43.596 & $0.830(0.785-0.874)$ & 0.066 & 33.3 & 7.4 \\
\hline & $\mathrm{TP}, \mathrm{mg} / \mathrm{L}$ & $U$ & 1481.6 & $0.547(0.500-0.593)$ & 0.070 & 0.0 & 8.2 \\
\hline & $\mathrm{NAG} / \mathrm{Cr}, \mathrm{U} / \mathrm{mmol} / \mathrm{L} \mathrm{Cr}$ & $U$ & 6.3 & $0.743(0.688-0.797)$ & 0.063 & 20.0 & 7.8 \\
\hline & $A L P, U / L$ & $U$ & 19 & $0.553(0.480-0.625)$ & 0.072 & 41.7 & 8.0 \\
\hline & GGT, U/L & $U$ & 198 & $0.538(0.465-0.610)$ & 0.071 & 40.0 & 7.7 \\
\hline & $\mathrm{LDH}, \mathrm{U} / \mathrm{L}$ & $\mathrm{S}$ & 238 & $0.779(0.741-0.818)$ & 0.102 & 37.8 & 15.2 \\
\hline & $\alpha 1 \mathrm{M}, \mathrm{mg} / \mathrm{L}$ & $U$ & 41.9 & $0.720(0.659-0.781)$ & 0.073 & 0.0 & 12.5 \\
\hline & $\beta 2 \mathrm{M}, \mathrm{mg} / \mathrm{L}$ & $U$ & 26.76 & $0.508(0.437-0.579)$ & 0.083 & 50.0 & 16.0 \\
\hline \multicolumn{8}{|l|}{ Clinical markers } \\
\hline \multicolumn{2}{|c|}{ Percentage of change in creatine } & & 0.31 & $0.627(0.578-0.677)$ & 0.098 & 19.6 & 10.7 \\
\hline \multicolumn{2}{|c|}{ eGFR at admission, $\mathrm{mL} / \mathrm{min} / 1.73 \mathrm{~m}^{2}$} & & 68.32 & $0.777(0.735-0.820)$ & 0.087 & 23.7 & 8.9 \\
\hline \multicolumn{2}{|l|}{$\mathrm{UP}, \mathrm{mL} / \mathrm{kg} / \mathrm{h}$} & & 0.92 & $0.728(0.680-0.777)$ & 0.092 & 26.4 & 9.8 \\
\hline \multicolumn{2}{|l|}{ APACHE II } & & 11 & $0.855(0.827-0.884)$ & 0.085 & 35.7 & 9.5 \\
\hline \multicolumn{2}{|l|}{ SOFA } & & 5 & $0.847(0.818-0.877)$ & 0.088 & 38.7 & 10.4 \\
\hline \multicolumn{2}{|l|}{ MODS } & & 3 & $0.843(0.813-0.873)$ & 0.087 & 30.0 & 10.7 \\
\hline
\end{tabular}

ACR, albumin-to-creatinine ratio; AKI, acute kidney injury; ALB, albumin; ALP, alkaline phosphatase; APACHE II, Acute Physiology and Chronic Health Evaluation II; $\mathrm{AUC}$, area under the receiver operating characteristic curve; $\mathrm{BUN}$, blood urea nitrogen; $\mathrm{Cl}$, confidence interval; $\mathrm{Cr}$, creatinine; CysC, cystatin C; eGFR, estimated glomerular filtration rate; GGT, gamma-glutamyl transpeptidase; IL-6, interleukin-6; LAC, lactic acid; LDH, lactate dehydrogenase; LOOCV, leave-one-out cross-validation; MODS, Multiple Organ Dysfunction Score; MSE, mean square error; NAG, $N$-acetyl- $\beta$-d-glucosaminidase; NAG/ $\mathrm{Cr}$, NAG-to-creatinine ratio; PCT, procalcitonin; S, serum; SOD, superoxide dismutase; SOFA, Sequential Organ Failure Assessment; TP, total protein; TRF, transferrin; $U$, urine; UP, urine production for the first $24 \mathrm{~h}$ after admission; $\alpha 1 \mathrm{M}, \alpha 1$-microglobulin; $\beta 2 \mathrm{M}, \beta 2$-microglobulin.

the correlation between CysC and LAC was relatively weak, with Spearman's rank correlation coefficient of only 0.0975. Consequently, the combination of $\mathrm{CysC}$ and $\mathrm{LAC}$ was a very good predictor for severe AKI, with an AUC of 0.907 , a positive predictive value (PPV) of 0.86 , and a negative predictive value (NPV) of 0.931 (Table 5). The values of AUC in predicting AKI and severe AKI by combining $\mathrm{Cys} \mathrm{C}$ or biomarkers or clinical markers are shown in Figure 2. Moreover, boxplots for each of the eventual outcomes of the AKI stage were created for $\mathrm{Cys} C$ and APACHE II score. We also determined that combinations of biomarkers or clinical markers markedly improved the prediction abilities of a single biomarker (Table 5).

\section{DISCUSSION}

In this study, we measured the abilities of 17 clinically available blood and urinary biomarkers to predict the occurrence of AKI and severe AKI in 1498 ICU patients. Ultimately, we found that three biomarkers, CysC, $\mathrm{ACR}$, and NAG/Cr, demonstrated significantly higher predictability in the severe AKI group than in the AKI 
Hou et al: : Assessment of 17 clinically available renal biomarkers to predict acute kidney injury in critically ill patients

\begin{tabular}{llllll}
\hline \multicolumn{7}{l}{ Table 4: Performances of biomarkers for total AKI and severe AKI } & & \\
\hline Biomarkers & \multicolumn{1}{l}{ Performance stratification } & AUC comparison & & \\
\cline { 2 - 5 } & Total AKI & Severe AKI & Total AKI & Severe AKI & $0.760(0.710-0.809)$ \\
\hline IL-6 & Fair & Fair & $0.710(0.665-0.756)$ & 0.145 \\
PCT & Useless & Useless & $0.595(0.560-0.629)$ & $0.619(0.575-0.663)$ & 0.331 \\
SOD & Useless & Useless & $0.464(0.418-0.511)$ & $0.516(0.449-0.583)$ & 0.177 \\
CysC & Fair & Good & $0.785(0.749-0.822)$ & $0.883(0.850-0.916)$ & 0.010 \\
BUN & Useless & Useless & $0.785(0.748-0.821)$ & $0.856(0.819-0.893)$ & 0.033 \\
TRF & Poor & Poor & $0.693(0.649-0.737)$ & $0.764(0.708-0.820)$ & 0.072 \\
LAC & Poor & Poor & $0.600(0.564-0.635)$ & $0.633(0.585-0.681)$ & 0.230 \\
Phosphate & Useless & Useless & $0.540(0.489-0.591)$ & $0.574(0.502-0.647)$ & 0.367 \\
ACR & Fair & Good & $0.759(0.722-0.796)$ & $0.835(0.793-0.877)$ & 0.032 \\
ALB & Useless & Useless & $0.727(0.686-0.768)$ & $0.830(0.785-0.874)$ & 0.016 \\
TP & Useless & Useless & $0.511(0.473-0.549)$ & $0.547(0.500-0.593)$ & 0.207 \\
NAG/Cr & Fair & Fair & $0.708(0.668-0.748)$ & $0.743(0.688-0.797)$ & 0.252 \\
ALP & Useless & Useless & $0.525(0.477-0.573)$ & $0.553(0.480-0.625)$ & 0.428 \\
GGT & Useless & Useless & $0.493(0.445-0.541)$ & $0.538(0.465-0.610)$ & 0.244 \\
LDH & Fair & Fair & $0.764(0.732-0.795)$ & $0.779(0.741-0.818)$ & 0.479 \\
$\alpha 1 \mathrm{M}$ & Poor & Poor & $0.652(0.603-0.700)$ & $0.720(0.659-0.781)$ & 0.099 \\
$\beta 2 M$ & Useless & Useless & $0.503(0.451-0.556)$ & $0.508(0.437-0.579)$ & 0.880 \\
\hline
\end{tabular}

Performances of biomarkers were stratified into useless, poor, fair, good, and excellent according to the AUC range of less than $0.60,0.60-0.69,0.70-0.79$, $0.80-0.89$, and $0.90-1.00$, respectively.

ACR, albumin-to-creatinine ratio; $\mathrm{AKI}$, acute kidney injury; $\mathrm{ALB}$, albumin; ALP, alkaline phosphatase; $\mathrm{AUC}$, area under the receiver operating characteristic curve; BUN, blood urea nitrogen; Cl, confidence interval; CysC, cystatin C; GGT, gamma-glutamyl transpeptidase; IL-6, interleukin-6; LAC, lactic acid; LDH, lactate dehydrogenase; NAG, $N$-acetyl- $\beta$-d-glucosaminidase; NAG/Cr, NAG-to-creatinine ratio; PCT, procalcitonin; SOD, superoxide dismutase; TP, total protein; TRF, transferrin; $\alpha 1 \mathrm{M}$, $\alpha 1$-microglobulin; $\beta 2 \mathrm{M}, \beta 2$-microglobulin.
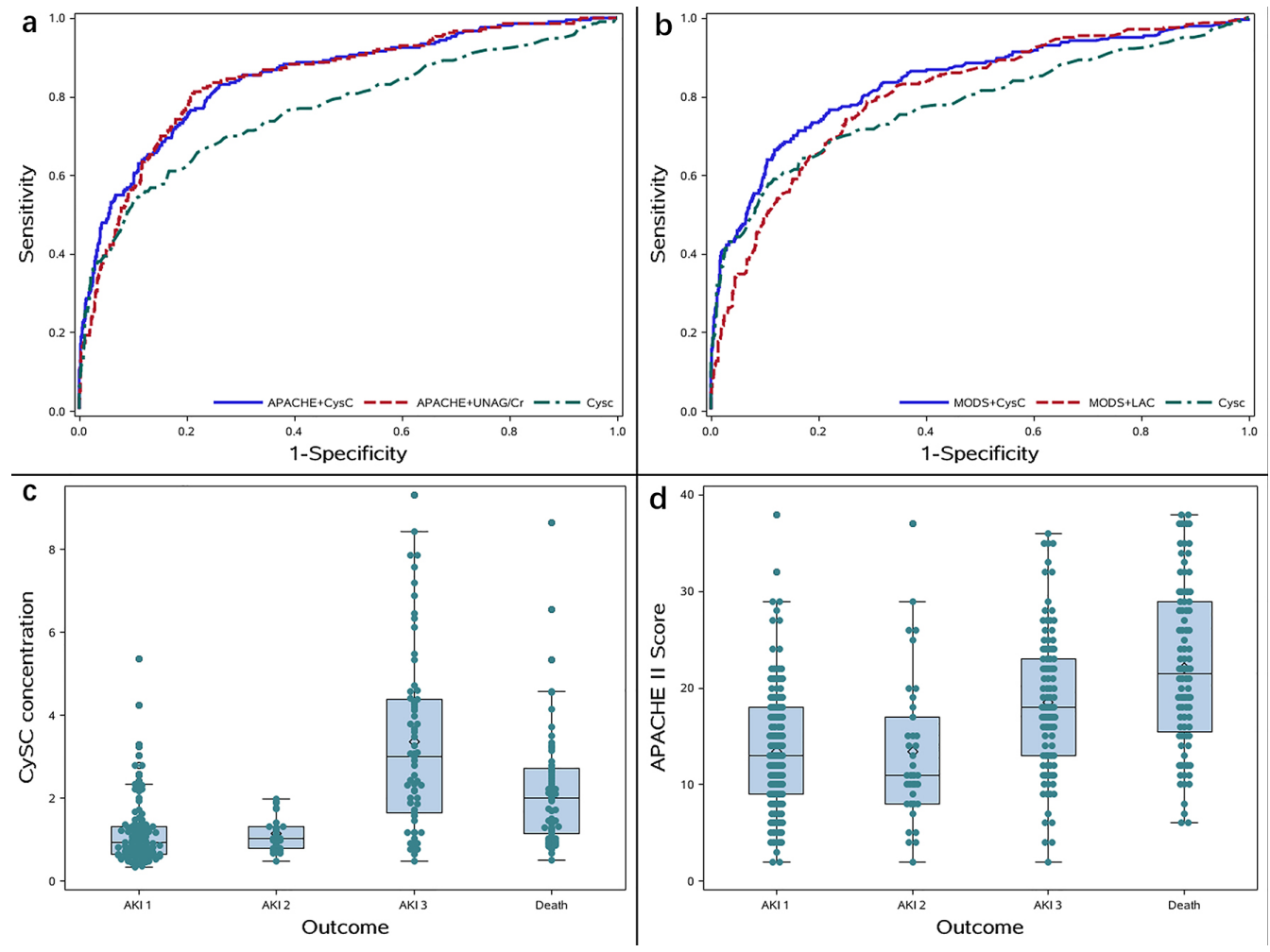

Figure 2: The best panel of combining CysC or biomarkers or clinical markers to predict the outcomes of AKI and severe AKI. (a) The AUCs for the panels of CysC, CysC and NAG/Cr, and CysC and APACHE II score to predict AKI were $0.785,0.856$, and 0.868 , respectively. (b) The AUCs for the panels of CysC, CysC and LAC, CysC and MODS to predict severe AKI were 0.883, 0.907, and 0.912, respectively. (c) Box and whisker plot for patients' CysC concentration value to predict AKI stage 1, 2, or 3 and death. (d) Box and whisker plot for patients' APACHE II score value to predict AKI stage 1, 2, or 3 and death. Boxes show the median value and 25th and 75th percentiles. Whiskers represent the range of values.

AUC: area under the receiver operating characteristic curve; AKI: acute kidney injury; APACHE II: Acute Physiology and Chronic Health Evaluation II; CysC: cystatin C; LAC: lactic acid; MODS: Multiple Organ Dysfunction Score; NAG: $N$-acetyl- $\beta$-d-glucosaminidase; NAG/Cr: NAG-to-creatinine ratio. 
Hou $e t$ al.: Assessment of 17 clinically available renal biomarkers to predict acute kidney injury in critically ill patients

\begin{tabular}{|c|c|c|c|c|c|c|}
\hline & AUC $(95 \% \mathrm{Cl})$ & MSE & Cutoff & PPV (95\% CI) & NPV (95\% CI) & $P$-value \\
\hline \multicolumn{7}{|l|}{ Total AKI } \\
\hline CysC + APACHE II & $0.868(0.831-0.886)$ & 0.106 & $0.407^{a}$ & $0.753(0.69-0.816)$ & $0.886(0.866-0.906)$ & $<0.0001$ \\
\hline $\mathrm{CysC}+\mathrm{NAG} / \mathrm{Cr}$ & $0.856(0.814-0.898)$ & 0.128 & $0.21^{\mathrm{a}}$ & $0.705(0.613-0.797)$ & $0.91(0.883-0.937)$ & 0.0034 \\
\hline CysC & $0.785(0.749-0.822)$ & 0.118 & $1.12 \mathrm{mg} / \mathrm{L}$ & $0.82(0.754-0.887)$ & $0.862(0.841-0.883)$ & Ref. \\
\hline \multicolumn{7}{|l|}{ Severe AKI } \\
\hline CysC + MODS & $0.912(0.883-0.941)$ & 0.057 & $0.488^{a}$ & $0.75(0.655-0.845)$ & $0.941(0.927-0.955)$ & 0.0015 \\
\hline CysC + LAC & $0.907(0.875-0.938)$ & 0.058 & $0.688^{\mathrm{a}}$ & $0.86(0.764-0.956)$ & $0.931(0.916-0.946)$ & 0.0129 \\
\hline CysC & $0.883(0.85-0.916)$ & 0.06 & $1.13 \mathrm{mg} / \mathrm{L}$ & $0.82(0.714-0.927)$ & $0.926(0.911-0.941)$ & Ref. \\
\hline
\end{tabular}

${ }^{a}$ Cutoff points of the biomarker panels were the predicted probabilities generated from the multiple logistic regression model.

AKI, acute kidney injury; APACHE II, Acute Physiology and Chronic Health Evaluation II; AUC, area under the receiver operating characteristic curve; $\mathrm{Cl}$, confidence interval; CysC, cystatin C; LAC, lactic acid; MSE, mean square error; MODS, Multiple Organ Dysfunction Score; NAG, $N$-acetyl- $\beta$ - $d$ glucosaminidase; NAG/Cr, NAG-to-creatinine ratio; NPV, negative predictive value; PPV, positive predictive value.

group. It is not surprising that most of the biomarkers are better at predicting severe AKI versus milder AKI, as severe $\mathrm{AKI}$ is a more homogeneous group. CysC showed the best predictive ability in AKI and severe AKI, with the highest AUC and the lowest MSE value, analogous to our previous studies. ${ }^{[33,34,37]}$

Similar to our prior studies, ${ }^{[34,37]}$ this study confirmed that a combination of biomarkers could improve the prediction ability for both $\mathrm{AKI}$ and severe AKI. For detecting AKI and severe $\mathrm{AKI}$, $\mathrm{Cys} C$ was regarded as a functional biomarker with the highest AUC and lowest MSE. NAG/Cr served as a tubular damage biomarker with relatively higher AUC and lower MSE. Thus, $\mathrm{CysC}$ and NAG/Cr combination had superior diagnostic performance in predicting AKI and severe AKI. We further found that the combination of biomarkers that could obviously improve prediction ability conforms to two characteristics. First, since the combination comprising a functional biomarker and a tubular damage biomarker can mirror distinct damage mechanisms of the nephron, this combination is superior. Additionally, serum and urine samples were contained in this panel. Second, the correlation between the combined biomarkers is small. This is because the smaller the correlation, the lesser the possibility of collinearity and the greater the possible combination benefit, such as in the combination of $\mathrm{CysC}$ and LAC. Similarly, a combination of geographically distant biomarkers in the cluster analysis dendrogram may also have a higher combination effect that improves prediction ability. Thus, the current study hinted that an alliance of diverse features and miscellaneous specimen sources (serum and urine) might be a rational policy for AKI diagnosis and prognosis in complex clinical situations.

According to the literature, ${ }^{[38]}$ adjustment of biomarkers for clinical factors can improve the predictive ability for
AKI. Due to the small number of patients involved in the prognostic investigations in previous studies, the CIs were large. ${ }^{[39-43]}$ The current study compared a more significant number of marker combinations between biomarkers and clinical markers in a large heterogeneous population to address this issue. Regarding the combinations of biomarkers and clinical factors, the panel of $\mathrm{Cys} C$ plus APACHE II score and the panel of CysC plus MODS were found to be the best panels in this cohort study for predicting AKI and severe AKI, respectively. One potential explanation for these results is that the clinical factors reflect the functions of other vital organs. The injury of these organs will directly or indirectly lead to renal function damage. Our findings also suggest that adhering clinical factors to biomarkers could generally improve the predictive performance, highlighting the complementarity between biomarkers and clinical markers and the significance of using biologic and clinical markers together to assess the functional status of organs.

Physicians are keen on evolving optimal measurements to quantify renal function independent of sCr or $\mathrm{UO}$ to detect AKI early and afford opportunities to advance clinical management. ${ }^{[4]]}$ Therefore, we expected that some of the existing markers would be routinely incorporated into AKI definition and diagnostics. Our study suggests that combining the current biomarkers and clinical markers can predict either AKI or severe AKI. Additionally, predictive markers could improve enrollment in clinical trials, allowing for the selection of patients at higher risk of adverse outcomes, who will most likely benefit from interventions, which would decrease the cost.

Both AUC, an indicator reflecting the degree of discrimination, and MSE, an indicator reflecting accuracy, were adopted to evaluate a particular marker's prediction 
efficiency comprehensively. We confirmed that an ideal marker should have a high AUC value and a low MSE value, which is consistent with the general statistical knowledge. Ultimately, we found that many biomarkers, such as PCT, were ineffective in predicting AKI or severe AKI. Though this result was not beyond expectation, we scientifically determined, for the first time, why these markers were ineffective, based on two aspects. First, some markers, such as urinary GGT, were ineffective because their apparent AUC was very small ( $<0.6$ or even lesser), which suggests that they have no specific ability. To our surprise, the AUC of serum BUN was even higher than CysC in critical conditions. However, it is not suitable as a biomarker because of its higher cutoff than its ULN. Consistently, CysC presented a robust performance in all our ways of assessment scale, irrespective of using MSE or AUC, regardless of its use solely or in combination with the clinical scoring system, including APACHE II score. These results encourage our clinicians and researchers to think outside the box and be aware that $\mathrm{CysC}$ is promising as a routine indicator for AKI to make up for the limitations of $\mathrm{sCr}$, which remains the renal functional indicator in the current clinic despite its many unacceptable disadvantages. Furthermore, the best combinations will depend on context and cohort. For instance, the combination of $\mathrm{Cys} C$ plus LAC performed well here, but it does not mean that all subjects with a normal LAC are protected from developing AKI.

Significantly, this is a large study with the potential to provide valuable information about many older, but potentially useful functional and damaged kidney biomarkers of AKI that preceded the era of novel damage biomarkers. Also of note, our article is the first to systematically explore the predictive capabilities of up to 17 clinically available biomarkers and several clinical markers from multiple perspectives, shifting our readers from "knowing which marker is useful or useless" to "knowing why this marker is useful or useless."

However, some limitations also existed in our study. First, some biomarkers were tested only once at ICU admission. Different biomarkers have their unique kinetics of concentration versus time after injury, and this kinetics may also change with changes in baseline GFR. Thus, simply measuring all biomarkers at once limits the performance of each biomarker and may mislead the viewer regarding the utility and accurate prediction of biomarkers. However, the KDIGO does not recommend a serialized detecting timetable because it is not maneuverable and cost-effective for gathering and testing sequences of samples at continual time points. Also, not only for an ethical reason, but also because it is difficult for patients and their family members to accept multiple blood and urine samples, the KDIGO does not recommend a serialized detecting timetable. Therefore, we focused on the level at ICU admission to initially assess the ability of clinically available biomarkers in predicting AKI and severe AKI. In the following study, we would further explore whether a series of measurements depending on the kinetic profile can improve prediction of AKI by a few promising biomarkers. Second, since $\mathrm{sCr}$ was lagging behind the actual renal injury and UO criteria were not used in predicting AKI, this survey likely missed many patients with AKI. Hence, the true prevalence of AKI might be affected. However, the incidence of AKI found in our study was basically the same as in previous studies, indicating that we had conservatively diagnosed the occurrence of AKI.

\section{CONCLUSION}

In this relatively large study of 1498 heterogeneous general ICU patients, $\mathrm{Cys} C$ demonstrated the best prediction power for AKI in the ICU, and its ability could be improved when combined with other biomarkers or clinical scores. Nevertheless, most clinically available biomarkers showed moderate performances in predicting AKI in critically ill patients.

\section{DECLARATIONS}

\section{Ethics approval and consent to participate}

The ethics committee of the Guangdong Provincial People's Hospital approved the protocol. Written informed consent was obtained from each patient or from appropriate surrogates for patients unable to consent.

\section{Competing interests}

The authors declare that they have no competing interests.

\section{Source of Funding}

The author Chunbo Chen is currently receiving a grant (\#number of MaoRenCaiBan[2020]24) from the Office of Talent Work Leading Group in Maoming, a grant (\#201803010058) from the Guangzhou Science and Technology Program, a grant (\#81671963) from the National Natural Science Foundation of China, and a grant (\#DFJH2020028) under the Major Program of Summit Project, Guangdong Province High-level Hospital Construction Project of Guangdong Provincial People's Hospital, Guangdong Academy of Medical Sciences. Author Linhui $\mathrm{Hu}$ is currently receiving a grant (\#2020YJ01) from the Emergent Science and Technology Project for Prevention and Treatment of Novel Coronavirus Pneumonia and a grant (\#zx2020017) from 
the High-level Hospital Construction Research Project of Maoming People's Hospital, and a grant (\#SY2021005) from Excellent Young Talents Project of Maoming People's Hospital. Author Feng Xu is currently receiving a grant (\#81873950) from the National Natural Science Foundation of China. Author Yujun Deng is presently receiving a grant (\#2016A020215129) from the Science and Technology Planning Project of Guangdong Province, China. The study was supported by the High-level Hospital Construction Research Project of Maoming People's Hospital.

\section{Acknowledgments}

The authors would like to thank all the doctors, nurses, technicians, and patients involved in the ICU for their dedication to the study.

\section{REFERENCE}

1. Hoste EA, Bagshaw SM, Bellomo R, Cely CM, Colman R, Cruz DN, et al. Epidemiology of acute kidney injury in critically ill patients: the multinational AKI-EPI study. Intensive Care Med 2015;41:1411-23.

2. Zhang D, Gao L, Ye H, et al. Impact of thyroid function on cystatin $\mathrm{C}$ in detecting acute kidney injury: a prospective, observational study. BMC Nephrol 2019;20:41.

3. Liang S, Shi M, Bai Y, et al. The effect of glucocorticoids on serum cystatin $\mathrm{C}$ in identifying acute kidney injury: a propensity-matched cohort study. BMC Nephrol 2020;21:519.

4. Ma J, Deng Y, Lao H, et al. A nomogram incorporating functional and tubular damage biomarkers to predict the risk of acute kidney injury for septic patients. BMC Nephrol 2021;22:176.

5. Deng Y, Yuan J, Chi R, et al. The Incidence, Risk Factors and Outcomes of Postoperative Acute Kidney Injury in Neurosurgical Critically Ill Patients. Sci Rep 2017;7:4245.

6. Yang L, Xing G, Wang L, et al. Acute kidney injury in China: a crosssectional survey. Lancet 2015;386:1465-71.

7. Martins CB, De Bels D, Honore PM, Redant S. Early Prediction of Acute Kidney Injury by Machine Learning: Should We Add the Urine Output Criterion to Improve this New Tool?. J Transl Int Med 2020;8:201-02.

8. van IJzendoorn $M$, de Vries $L$, van den Born J, Buter H, Navis G, Boerma C. Renal Function is a Major Determinant of ICU-acquired Hypernatremia: A Balance Study on Sodium Handling. J Transl Int Med 2020;8:165-76.

9. Lang R, Wang X, Liang Y, Yan L, Shi B, Yu R. Research Progress in the Treatment of Idiopathic Membranous Nephropathy using Traditional Chinese Medicine. J Transl Int Med 2020;8:3-8.

10. Wu Y, Peng W, Wei R, et al. Rat mRNA expression profiles associated with inhibition of ischemic acute kidney injury by losartan. Biosci Rep 2019;39:BSR20181774.

11. Fang M, Liu S, Zhou Y, et al. Circular RNA involved in the protective effect of losartan on ischemia and reperfusion induced acute kidney injury in rat model. Am J Transl Res 2019;11:1129-44.

12. Deng Y, Chi R, Chen S, et al. Evaluation of clinically available renal biomarkers in critically ill adults: a prospective multicenter observational study. Crit Care 2017;21:46.

13. Devarajan P. Biomarkers for the early detection of acute kidney injury. Curr Opin Pediatr 2011;23:194-200.

14. Koyner JL, Vaidya VS, Bennett MR, et al. Urinary biomarkers in the clinical prognosis and early detection of acute kidney injury. Clin J Am
Soc Nephrol 2010;5:2154-65.

15. Ostermann M, Joannidis M. Acute kidney injury 2016: diagnosis and diagnostic workup. Crit Care 2016;20:299.

16. Khawaja S, Jafri L, Siddiqui I, Hashmi M, Ghani F. The utility of neutrophil gelatinase-associated Lipocalin (NGAL) as a marker of acute kidney injury (AKI) in critically ill patients. Biomark Res 2019;7:4.

17. Han WK, Bailly V, Abichandani R, Thadhani R, Bonventre JV. Kidney Injury Molecule-1 (KIM-1): a novel biomarker for human renal proximal tubule injury. Kidney Int 2002;62:237-44.

18. Fontanilla J, Han WK. Kidney injury molecule- 1 as an early detection tool for acute kidney injury and other kidney diseases. Expert Opin Med Diagn 2011;5:161-73.

19. Parikh CR, Abraham E, Ancukiewicz M, Edelstein CL. Urine IL-18 is an early diagnostic marker for acute kidney injury and predicts mortality in the intensive care unit. J Am Soc Nephrol 2005;16:3046-52.

20. Yang X, Chen C, Tian J, Zha Y, Xiong Y, Sun Z, et al. Urinary Angiotensinogen Level Predicts AKI in Acute Decompensated Heart Failure: A Prospective, Two-Stage Study. J Am Soc Nephrol 2015;26:2032-41.

21. Cui S, Wu L, Feng X, Su H, Zhou Z, Luo W, et al. Urinary angiotensinogen predicts progressive chronic kidney disease after an episode of experimental acute kidney injury. Clin Sci (Lond) 2018;132:2121-33.

22. Yang X, Ou J, Zhang H, Xu X, Zhu L, Li Q, et al. Urinary Matrix Metalloproteinase 7 and Prediction of IgA Nephropathy Progression. Am J Kidney Dis 2020;75:384-93.

23. Yang X, Chen C, Teng S, Fu X, Zha Y, Liu H, et al. Urinary Matrix Metalloproteinase-7 Predicts Severe AKI and Poor Outcomes after Cardiac Surgery. J Am Soc Nephrol 2017;28:3373-82.

24. Adler C, Heller T, Schregel F, Hagmann H, Hellmich M, Adler J, et al. TIMP-2/IGFBP7 predicts acute kidney injury in out-of-hospital cardiac arrest survivors. Crit Care 2018;22:126.

25. Ostermann M, Chang RW. Challenges of defining acute kidney injury. QJM 2011;104:237-43.

26. Chen C, Yang X, Lei Y, Zha Y, Liu H, Ma C, et al. Urinary Biomarkers at the Time of AKI Diagnosis as Predictors of Progression of AKI among Patients with Acute Cardiorenal Syndrome. Clin J Am Soc Nephrol 2016;11:1536-44.

27. Murray PT, Mehta RL, Shaw A, Ronco C, Endre Z, Kellum JA, et al. Potential use of biomarkers in acute kidney injury: report and summary of recommendations from the 10th Acute Dialysis Quality Initiative consensus conference. Kidney Int 2014;85:513-21.

28. Disease K. Kidney Disease: Improving Global Outcomes (KDIGO) Acute Kidney Injury Work Group. Kidney Int 2012;2:1-138.

29. von Elm E, Altman DG, Egger M, Pocock SJ, Gotzsche PC, Vandenbroucke JP, et al. The Strengthening the Reporting of Observational Studies in Epidemiology (STROBE) statement: guidelines for reporting observational studies. Ann Intern Med 2007;147:573-7.

30. Bossuyt PM, Reitsma JB, Bruns DE, Gatsonis CA, Glasziou PP, Irwig LM, et al. Toward complete and accurate reporting of studies of diagnostic accuracy: the STARD initiative. Acad Radiol 2003;10:664-9.

31. Kidney Disease Improving Global Outcomes (KDIGO) Acute Kidney Injury Work Group. KDIGO clinical practice guideline for acute kidney injury. Kidney Int Suppl 2012;2:1-138.

32. Zarbock A, Kellum JA, Schmidt C, Van Aken H, Wempe C, Pavenstädt H, et al. Effect of Early vs Delayed Initiation of Renal Replacement Therapy on Mortality in Critically Ill Patients With Acute Kidney Injury: The ELAIN Randomized Clinical Trial. Jama 2016;315:2190-9.

33. Wang L, Deng Y, Zhai Y, et al. Impact of blood glucose levels on the accuracy of urinary $\mathrm{N}$-acety- $\beta$ - $\mathrm{D}$-glucosaminidase for acute kidney injury detection in critically ill adults: a multicenter, prospective, observational study. BMC Nephrol 2019;20:186.

34. Deng Y, Wang L, Hou Y, et al. The influence of glycemic status on the performance of cystatin $\mathrm{C}$ for acute kidney injury detection in the critically ill. Ren Fail 2019;41:139-49.

35. Haase-Fielitz A, Bellomo R, Devarajan P, Story D, Matalanis G, Dragun 
D, et al. Novel and conventional serum biomarkers predicting acute kidney injury in adult cardiac surgery--a prospective cohort study. Crit Care Med 2009;37:553-60.

36. Hastie T, Tibshirani R, Friedman J. The Elements of Statistical Learning Data Mining, Inference, and Prediction. 2nd ed. The Elements of Statistical Learning Data Mining, Inference, and Prediction. New York, NY, USA: Springer; 2009.

37. Deng Y, Ma J, Hou Y, et al. Combining Serum Cystatin C and Urinary N-Acetyl-Beta-D-Glucosaminidase Improves the Precision for Acute Kidney Injury Diagnosis after Resection of Intracranial Space-Occupying Lesions. Kidney Blood Press Res 2020;45:142-56.

38. Koyner JL, Garg AX, Coca SG, Sint K, Thiessen-Philbrook H, Patel UD, et al. Biomarkers predict progression of acute kidney injury after cardiac surgery. J Am Soc Nephrol 2012;23:905-14.

39. Houde F, Laroche S, Thivierge V, et al. Transcranial Magnetic Stimulation Measures in the Elderly: Reliability, Smallest Detectable Change and the
Potential Influence of Lifestyle Habits. Front Aging Neurosci 2018;10:379.

40. Herr W, Krumkamp R, Hogan B, Dekker D, Gyau K, Owusu-Dabo E, et al. A cross-sectional study on risk factors for infection with Parvovirus B19 and the association with anaemia in a febrile paediatric population in Ghana. Sci Rep 2020;10:15695.

41. Lin M, Chen J, Li S, Qin Y, Wang X, Liu Y, et al. Risk factors for suicidal tendency in people with epilepsy in China: a case-control study. Sci Rep 2021;11:2742.

42. Platt RW. Introduction to statistics--1. The confidence interval. Inj Prev 1997;3:213.

43. Curran-Everett D. Explorations in statistics: confidence intervals. Adv Physiol Educ 2009;33:87-90.

44. Endre ZH, Kellum JA, Di Somma S, et al. Differential diagnosis of AKI in clinical practice by functional and damage biomarkers: workgroup statements from the tenth Acute Dialysis Quality Initiative Consensus Conference. Contrib Nephrol 2013;182:30-44.

How to cite this article: Hou Y, Deng $Y$, Hu L, He L, Yao F, Wang $Y$, et al. Assessment of 17 clinically available renal biomarkers to predict acute kidney injury in critically ill patients. J TransI Intern Med 2021; 9 : 273-84. 